\title{
Validation of SICK score - A severity of illness scoring system at a tertiary care centre in Puducherry
}

\author{
Babji S.K ${ }^{1}$, Bala gopal $M^{2}$, Sharma N.A ${ }^{3}$, Sivaguru $C^{4}$ \\ ${ }^{1}$ Dr. Shivaramakrishna Babji N., Assistant Professor, Department of pediatrics, Sri Manakula Vinayagar Medical \\ College, Puducherry, ${ }^{2}$ Dr. Bala gopal. M, Associate Professor, Department of Pediatrics, Sri Manakula Vinayagar \\ Medical College, Puducherry, ${ }^{3}$ Dr. N Anoop Sharma, Ex Registrar, Saint Stephen Hospital, New Delhi, ${ }^{4}$ Dr. Chandran \\ Sivaguru, Ex Specialist Grade 1 (pediatrics) and Head of the Department, Indira Gandhi Government General Hospital \& \\ Post Graduate Institute, Puducherry, India.
}

Author for Correspondence: Dr. N. Shivaramakrishna Babji, No. 29, Assistant Professor Quarters, SMVMCH Campus, Kalitheerthal Kuppam, Puducherry, E mail: shivababji@gmail.com

\begin{abstract}
Objectives: Validation of SICK score for its ability to predict out come. Validation based on predominant system involved. Methods: Prospective study done at tertiary care centre in puducherry. Inclusion criteria: All children admitted in PICU between 1 month \& 12 year in 6 month period. Exclusion criteria: Patients with surgical illness, congenital anomalies, non availability of consent. Physical variables of Temperature, Heart Rate, Respiratory Rate, Blood pressure, Capiallary Refill time, Oxygen saturation \& sensoriurn (using AVPU scale) were recorded at the time of admission. SICK score was calculated and outcome was recorded as survived or expired. Association of patients outcome with total score was done using logistic regression analysis. The receiver operating characteristic (ROC) curve analysis was performed to decide the optimum cut off point in terms of total score that provides maximum sensitivity \& specificity. Area under ROC curve (AUC) was used to compare the predictive power of total score. All analysis was carried out using SPPS software version 13.0. Results: Total of 354 Children were analyzed. For SICK score of $<1$ mortality was $0 \%$, which gradually increased to $100 \%$ with a score of 7 or more. The area under the ROC curve was 93\% (95\%: CI: $89.8 \%$ - 97.3\%) indicating good predictive ability of the score. Conclusion: SICK score has good predictive ability and uses only physical criteria. Further SICK score is not influenced by predominant system involved.
\end{abstract}

Key words: Physical variables, SICK score, Outcome

\section{Introduction}

Mortality in an intensive care unit depends on severity of illness and quality of care accordingly [1]. Severity of illness scores in pediatrics like the PRISM Score (Pediatric Risk of Mortality Score) and PIM (Pediatric Index of Mortality Score) serve the purposes of comparative audit (comparing actual with expected outcomes over different units), and research (adjusting for differences in the case mix).

These scoring systems could be used as a tool to triage, prioritize care and obviate harmful delay in the management of sick children [2]. Mortality depends on the quality of care received in the first 24 hours of being ill [3]. However many of the scoring systems depend on

Manuscript received: $10^{\text {th }}$ March 2017

Reviewed: $17^{\text {th }}$ March 2017

Author Corrected: $24^{\text {th }}$ March 2017

Accepted for Publication: $31^{\text {st }}$ March 2017 both physical and laboratory variable, take $24 \mathrm{hrs}$ or more for predicting outcome. Hence they are inappropriate for primary triage and are cost and labor intensive $[1,4]$. This window of opportunity of treating the child aggressively is lost by the time PRISM and PIM scores are available [5].

ETAT (Emergency Triage, Assessment and Treatment) score developed by WHO \& requires reorganization of existing health care system. For assessing ETAT score, special training is required for staff and doctors [6,7]. A study by Thompson $\mathrm{N}$ et al. concluded that a combination of vital signs can be used to differentiate children based on severity of sickness with a comparable sensitivity to more complicated triage systems [8]. SICK Score, a scoring system developed and validated is based only on physical variables of 
SIRS (systemic inflammatory response syndrome) and MODS (multi organ dysfunction syndrome) as a practical Triage tool at the time of first contact for predicting the severity of sickness and mortality [9]. This scoring system has been validated in the UK and at a center in Delhi [4], where it originated but has not been tested in other centers in India. Hence, we set out to validate the scoring system in a Tertiary Care hospital in Puducherry. The validity is being tested primarily to look at its ability to predict outcome and to further evaluate the validity of this score based on the predominant system involved at the time of presentation which was not found to be done previously.

\section{Materials and Methods}

This is prospective study done at Indira Gandhi Government General Hospital \& Postgraduate Institute, Pondicherry, tertiary care center. Ethical clearance was obtained from the Institutional Ethics Committee. Written informed consent was obtained from parents of all the children for participation in the study and analysis of their vital parameters.

\section{Inclusion criteria}

All the children admitted consecutively in PICU in the age group of 1 month to 12 years between January 2008 and June 2008 were enrolled in the study.

\section{Exclusion criteria}

Children with surgical problems, congenital anomalies, those discharged against medical advice and those who did not give consent were excluded.

Physical variables of temperature, heart rate, respiratory rate, blood pressure, capillary refill time (CRT), oxygen saturation (pulse oximetry) and sensorium (using AVPU scale) were recorded the time of admission as per the SICK scoring system (as shown in Table 1). Temperature was measured in unilateral axilla using digital thermometer. Heart rate was measured by auscultatory method and respiratory rate was measured by observation for a period of $1 \mathrm{~min}$. Blood pressure was measured using mercury sphygmomanometer of appropriate cuff size. CRT was measured by lifting the lower limb slightly above the heart level and applying pressure over great toe for 5 seconds and then counting the time in seconds for capillary refill and the consciousness was ascertained using AVPU score. Each variable in the study was scored as 0 (zero) for being normal and score of 1 for being abnormal and the SICK score was obtained. Duration of hospital stay, diagnosis, sex and age were also noted. Outcome was recorded as survived or expired.

Tabl-1: Chart used for assessment of Vital signs.

\begin{tabular}{|c|c|}
\hline Vital Sign & Abnormal \\
\hline Heart rate & $>160$ ( $<1$ year) \\
& $>150$ ( $>1$ year) \\
\hline Respiratory rate & $>60$ for $<2$ months \\
& $>50$ for 2 to 12 months \\
& $>40$ for $1-5$ years \\
& $>30$ for $6-12$ years \\
& Any gasping respiration \\
\hline Temperature & $>38^{\circ} \mathrm{C}$ \\
& $<36^{\circ} \mathrm{C}$ \\
\hline Blood pressure (Systolic) & $<70 \mathrm{~mm} \mathrm{Hg}(<1 \mathrm{month}$ to 1 year) \\
& $<$ age $2+70$ (1-10years) \\
& $<90 \mathrm{~mm}$ Hg $(>10$ years) \\
\hline
\end{tabular}


Original Research Article

\begin{tabular}{|c|c|}
\hline Capillary refill time & $>3$ seconds \\
\hline Pulse oximetry saturation & $<90 \%$ \\
\hline Sensorium level & Responsive to voice \\
(AVPU score) & Responsive to pain \\
Unresponsive
\end{tabular}

Statistical analysis: Scores were calculated using the online SICK score calculator available at http://jacob.puliyel.com/sick.php. Heterogeneity chi square test and Fisher's exact test were used to test the association of outcome (discharged or expired) with the physical variables. $\mathrm{Z}$ test of proportion is used to find the association between age and mortality in various age groups. Logistic regression analysis was done to examine the association of patient's outcome with total score.

The receiver operating characteristic (ROC) curve analysis was performed to decide the optimum cut-off point in terms of total score that provides maximum sensitivity (true positive rate, classification of those expired as expired by $>80 \%$ ) and specificity (true negative rate, classification of those discharged as discharged by $>80 \%$ ). The optimum cut-off point was defined as the closest point on the ROC curve to the point $(0,100)$ i.e., false positive rate (1-specificity) of zero and sensitivity of $100 \%$. The areas under the ROC curves (AUCs) and 95\% exact binomial confidence interval (CI) were used to compare the predictive power of total score that could classify the patients those who need critical care and those who can be discharged. In all the analysis a probability value of $<0.05$ was considered significant. All analysis were carried out using SPSS software version 13.0.

\section{Results}

Total of 375 children who met the inclusion criteria were recruited into the study. 21 children were excluded during follow up because of 5 surgical problems, 4 referral to higher centre and 12 discharged against medical advice. Total of 354 children were analyzed as final study group. Of them 39 children (11\%) expired in the hospital and the remaining 315 children (89\%) survived. Z - Test of proportion between two samples of the children in the study.

As $\mathrm{Z}$ score calculated is more than $\mathrm{Z}$ score table value (Table 2), we conclude that proportion of deaths is statistically significant in age group of $<5 \mathrm{yrs}$ with a $\mathrm{P}$ value of 0.05 .

Table-2: Age related mortality.

\begin{tabular}{|c|c|}
\hline First sample & Second sample \\
\hline$<5 y r s$ & $>5-12 \mathrm{yrs}$ \\
\hline 33 deaths out of 263 cases & 6 deaths out of 91 cases \\
\hline Proportion of deaths $\left(\mathrm{P}_{1}=0.125\right)$ & Proportion of deaths $\left(\mathrm{P}_{2}=0.065\right)$ \\
\hline
\end{tabular}

Hence we conclude that there is higher mortality in younger children. Of the total children 143 (40\%) are female and 211 $(60 \%)$ were male. A total of $13(9.1 \%)$ children among female and $26(12.3 \%)$ among male expired. Statistical analysis revealed that mortality was independent $\left(\chi^{2}=0.9, \mathrm{P}=0.3\right)$ of gender distribution.

Table 3 denotes the association of each of the physical variables with the outcome. The difference in the mortality rates between those in normal and abnormal range of temperature was not significant $\left(\chi^{2}=0.52, \mathrm{P}=0.47\right)$ indicating that mortality was independent of temperature. The odds of death was 1.3 (95\% CI: 0.6-2.7) times higher for the patients with abnormal temperature than those with the normal. The observed difference in the mortality rates between those with normal and abnormal heart rates was not significant $\left(\chi^{2}=0.03, \mathrm{P}=0.9\right)$.

The odds of death was 1.1 (95\% CI: 0.5-2.2) times higher for patients with abnormal heart rate than those with normal. The mortality rates differed significantly between those with normal and abnormal respiratory rates $\left(\chi^{2}=3.99, \mathrm{P}=0.045\right)$. 
The odds of death was 2.4 (95\% CI: 1.0-7.4) times higher for patients with abnormal respiration than those with normal. The mortality rates differed significantly between subjects with normal and abnormal blood pressure (Fisher's exact test, $\mathrm{P}<0.0001)$.

The odds of death was 6.9 (95\% CI: 3.0-15.9) times higher for patients with abnormal blood pressure than those with normal. The mortality rates differed significantly between patients with normal and abnormal CRT $\left(\chi^{2}=34.6, \mathrm{P}<0.0001\right)$. The odds of death was 7.1 (95\% CI: 3.2-15.5) times higher for patients with abnormal CRT than those with normal. The mortality rates differed significantly between patients with normal and abnormal $\mathrm{SPO}_{2}$ (Fisher's exact test, $\mathrm{P}<0.0001$ ).

Table-3: Association of variables and outcome.

\begin{tabular}{|c|c|c|c|c|}
\hline variable & & Survived No. \& \% & Died No. \& \% & P - Value \\
\hline \multirow[t]{2}{*}{ Temperature } & Normal & $212(89.8 \%)$ & $24(10.2 \%)$ & \multirow[t]{2}{*}{0.47} \\
\hline & Abnormal & $103(87.3 \%)$ & $15(12.7 \%)$ & \\
\hline \multirow[t]{2}{*}{ Heart Rate } & Normal & $134(89 . \%)$ & $16(10.7 \%)$ & \multirow[t]{2}{*}{0.9} \\
\hline & Abnormal & $181(88.7 \%)$ & $23(11.3 \%)$ & \\
\hline \multirow[t]{2}{*}{ Respiratory Rate } & Normal & $97(94.2 \%)$ & $6(5.8 \%)$ & \multirow[t]{2}{*}{0.045} \\
\hline & Abnormal & $218(86.9 \%)$ & $33(13.1 \%)$ & \\
\hline \multirow[t]{2}{*}{ Blood Pressure } & Normal & $289(92.3 \%)$ & $24(7.7 \%)$ & \multirow[t]{2}{*}{$<0.0001$} \\
\hline & Abnormal & $26(63.4 \%)$ & $15(36.6 \%)$ & \\
\hline \multirow[t]{2}{*}{ Capillary Refill Time } & Normal & $281(93 \%)$ & $21(7 \%)$ & \multirow[t]{2}{*}{$<0.0001$} \\
\hline & Abnormal & $34(65.4 \%)$ & $18(34.6 \%)$ & \\
\hline \multirow[t]{2}{*}{ SPO2 } & Normal & $298(96.4 \%)$ & $11(3.6 \%)$ & \multirow[t]{2}{*}{$<0.0001$} \\
\hline & Abnormal & $17(37.8 \%)$ & $28(62.2 \%)$ & \\
\hline \multirow{2}{*}{$\begin{array}{c}\text { Sensorium } \\
\text { (AVPU) }\end{array}$} & Normal & $184(97.9 \%)$ & $4(2.1 \%)$ & \multirow[t]{2}{*}{$<0.0001$} \\
\hline & Abnormal & $131(78.9 \%)$ & $35(21.1 \%)$ & \\
\hline
\end{tabular}

The odds of death was 44.6 (95\% CI: 17.6-114.9) times higher for patients with abnormal $\mathrm{SPO}_{2}$ than those with normal. The mortality rates differed significantly between the patients with normal and abnormal AVPU $\left(\chi^{2}=32.3, \mathrm{P}<0.0001\right)$.

The odds of death was 12.3 (95\% CI: 4.2-48.5) times higher for patients with abnormal AVPU than those with normal.

Variables measured were analyzed for association with the outcome (table 3). Table 4 denotes the percentage of mortality at different SICK score. With a SICK score of $<1$ mortality was $0 \%$, which gradually increased to $100 \%$ with a score of 7 or more (Table 4). Various cut off values were tried to categorize patients. Maximum discrimination was observed for a score of 3.75 , where sensitivity was $87 \%$ and specificity was $89 \%$. The differences between the observed and expected number of deaths \& survivals was not statistically significant. Homer-Lemeshaw Chi-square with 8 degrees of freedom was $4.125(\mathrm{p}=0.8)$. The ROC curve developed using different cut off points had 93\% (95\% CI: 89.8\%-97.3\%) of area under curve, indicating good predictive ability of the score(figure 1).

Table-4: Mortality with different SICK score.

\begin{tabular}{|c|c|c|c|c|}
\hline SICK score & Improved & Expired & Total & Mortality (\%) \\
\hline 0 & 09 & 00 & 09 & 00 \\
\hline$<1$ & 63 & 00 & 63 & 00 \\
\hline
\end{tabular}


Original Research Article

\begin{tabular}{|c|c|c|c|c|}
\hline $1-1.9$ & 54 & 01 & 55 & 1.8 \\
\hline $2-2.9$ & 109 & 00 & 109 & 00 \\
\hline $3-3.9$ & 50 & 05 & 55 & 09 \\
\hline $4-4.9$ & 22 & 16 & 38 & 42 \\
\hline $5-5.9$ & 07 & 06 & 13 & 46 \\
\hline $6-6.9$ & 01 & 06 & 07 & 100 \\
\hline$\geq 7$ & 00 & 05 & 05 & $\mathbf{3 5 4}$ \\
\hline Total & $\mathbf{3 1 5}$ & $\mathbf{3 9}$ & & \\
\hline
\end{tabular}

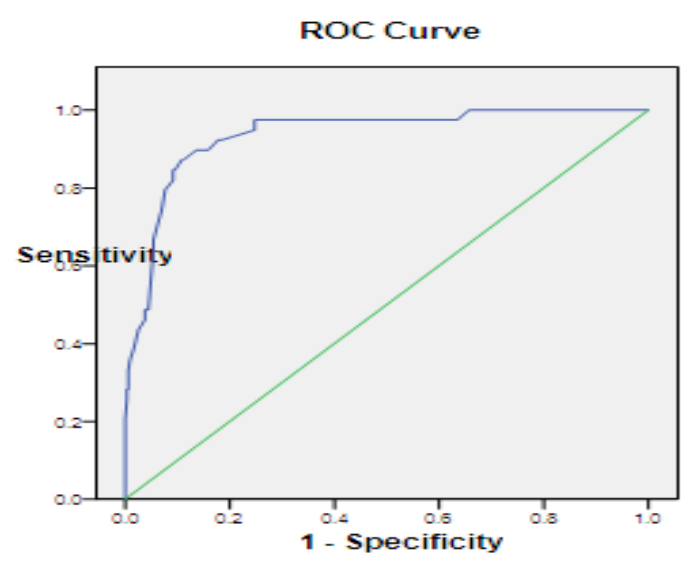

Diagonal segments are produced by ties.

Figure-1: SICK Score ROC Curve in this study (Area under ROC curve=0.936).

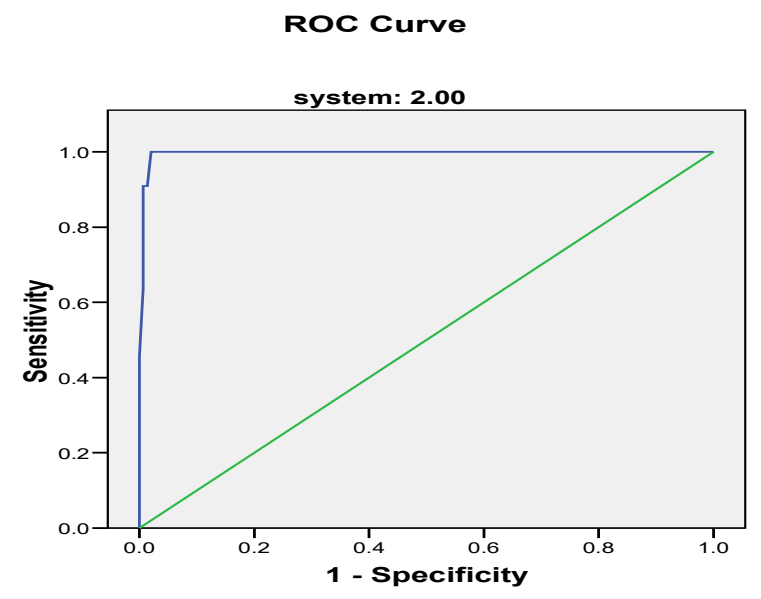

Figure-3: ROC curve for Respiratory system involvement (AUC IS 0.996)
ROC Curve

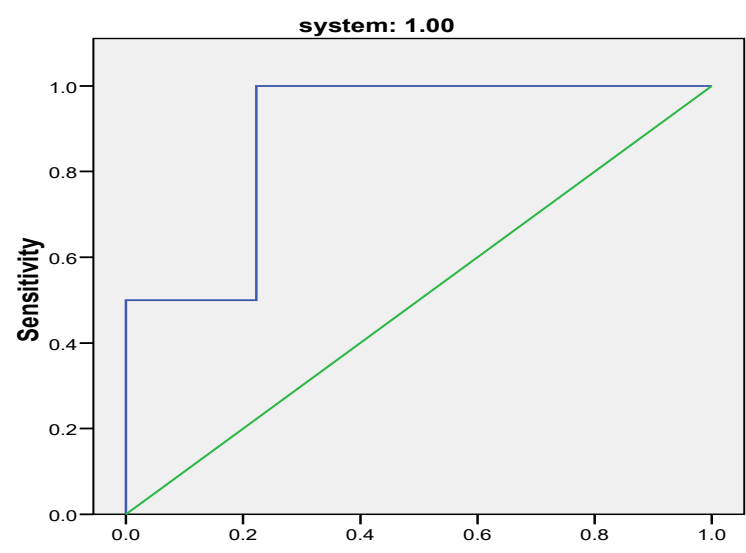

1-specificity

Figure-2: ROC Curve for GIT system involvement (AUC is 0.889)

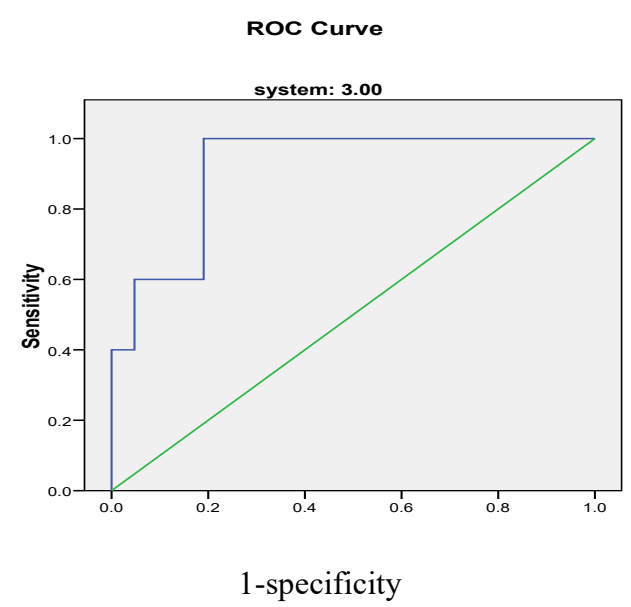

Figure-4: ROC Curve for Cardiovascular system (AUC is0.914). 

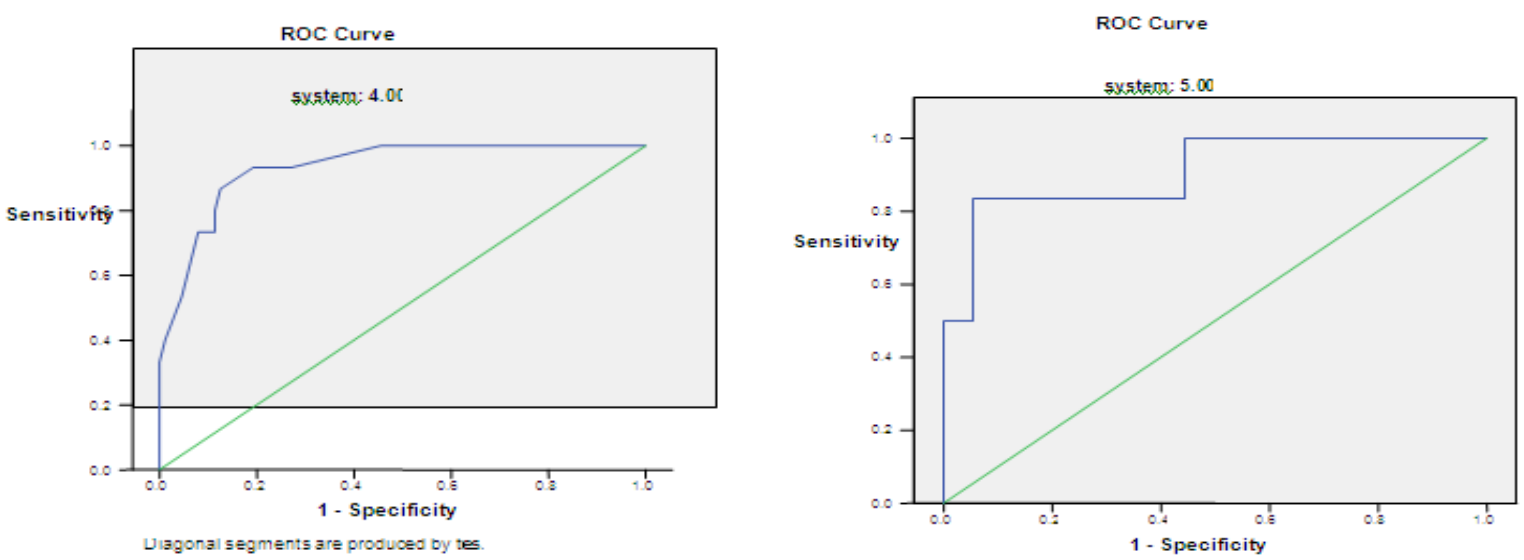

Figure-5: ROC curve for CNS (AUC is 0.933). Figure-6: ROC curve for other systems (AUC is 0.907).

The predictive ability of SICK score was also evaluated for assessing system wise mortality. Figures 2-6 show the area under ROC for predicting system wise mortality, indicating good predictive ability of SICK score across various organ systems.

\section{Discussion}

In a emergency department large number of patients visit every day. In a crowded emergency department consulting patients in the order of attending will lead to long waiting times, which may be detrimental for seriously ill patients [7]. Initial triage of sick children arriving at hospital in developing countries is often deficient with severely ill children experiencing days in the institution of life saving management [7].

The aim of triage is to determine \& classify the clinical priority of patients visiting the emergency department [10]. Triage in pediatric emergency care is an important tool to prioritize seriously ill children. Triage can also be used to identify patients who need urgent care and who can safely wait [11].

Early identification of severity of illness helps in prioritizing children, based on sickness for treatment to reduce mortality \& allow proper utilization of limited resources of the developing world. Most of the scoring system in pediatrics serves the purpose of comparative audit (comparing actual with expected outcomes over different units) and evaluative research (adjusting for differences in case mix) [8]. Few of the standard scoring systems like PRISM \& PIM over estimate the risk of mortality $[12,13,14]$.

A study done in Intensive care unit in south Africa has shown poor discriminatory performance of the PRISM at all age groups and diagnostic categories. SICK Scoring System based on physical variables alone, can be done at the time of first contact, helps in prioritizing children for treatment based on sickness. A combination of vital signs can be used to differentiate children with serious infections from those with less serious infections in a pediatric assessment unit and has comparable sensitivity to more complicated triage systems [8].

- Hence there is a need of scoring system.

- Which utilizes physical criteria alone

- Does not need special training for both staff and doctors

- Study by Pollack et al; concluded similar relationship exists between physiological instability and across different intensive care units [3].

Systemic inflammatory Response syndrome is a syndrome that encompasses the features of systemic inflammation as a response of the Immune system to an insult. SIRS is nonspecific and can be caused by ischemia, inflammation, trauma, infection or a combination of several insults[15].when SIRS is mediated by infectious or noninfectious insult, there is release of IL-1(Interleukin-1) and TNF $\alpha$ (Tumour necrosis factor alpha) which lead to cleavage of nuclear factor- $\kappa \mathrm{B}$ (NF-KB) inhibitor. All these lead to endothelial damage, microvascular thrombosis, increased vascular permeability leading to multi organ failure and death. 
Considering the high specificity of primary SIRS for admission (89.5\%) and mortality (86.8\%), SIRS criteria have clinical and prognostic importance in the management of emergency patients [16].

SIRS Criteria in children [17]: Presence of at least 2 of the following 4 criteria, 1 of which must be abnormal temperature or leukocyte count.

Core temperature of $>38.5^{\circ} \mathrm{C}$ or $<36^{\circ} \mathrm{C}$.

Tachycardia defined as mean heart rate $>2 \mathrm{SD}$ above normal for age in the absence of external stimulus, long term drug use or painful stimuli or otherwise unexplained persistent elevation over a 0.5 to $4 \mathrm{hr}$ period or for children $<1 \mathrm{yr}$ old:

Bradycardia, defined as a mean heart rate $<10^{\text {th }}$ percentile for age in the absence of external vagus stimulus, $\beta$-blocker drugs, or congenital heart disease; or otherwise unexplained persistent depression over a $0.5 \mathrm{hr}$ period.

Mean respiratory rate more than 2 SD above normal for age or mechanical ventilation for an acute process not related to underlying neuromuscular disease or the receipt of general anaesthesia.

Leukocyte count elevated or depressed for age (not secondary to chemotherapy induced leucopenia) or $>10 \%$ immature neutrophils.

When a sick child is brought to the Emergency department then rapid assessment can be done as per APLS [18]:

Airway or Breathing

- Effort of breathing

- Respiratory rate or rhythm

- Stridor / Wheeze

- Auscultation

- Skin colour

\section{Circulation}

- Heart rate

- Pulse volume

- Capillary refill

- Skin temperature

\section{Disability}

- Mental status or conscious level

- Posture

- Pupils

- The whole assessment should take less than 1 minute.
We tried to validate SICK score, a scoring system based on physical variables alone based on SIRS \& APLS guidelines.

Temperature is an important clinical variable in SIRS criteria in children. Temperature instability is one of the variables in APACHE score, which predicts the probability of death. Mc carthy PL et al states that high fever alone can be used to indicate bactaermia [19]. Heart rate is important component in assessment of cardiovascular system. Heart rate is an important clinical variable in SIRS criteria as well as APACHE and PRISM score to predict the mortality. Respiratory rate is part of the PRISM score which can predict mortality in PICU. In many studies tachypnoea has been found to be the most reliable clinical sign in a child who has pneumonia rather than upper respiratory tract infection $[20,21,22,23]$. Study done by Campbell $\mathrm{H}$ et al suggested that in children aged 1 to 4 years, fever $>38.5^{\circ} \mathrm{C}$ or respiratory rate $>60$ per minute indicates severe lower respiratory tract infection [21].

Blood pressure is an important parameter in the assessment of circulation. Blood pressure is also an important variable of PRISM score used to predict the mortality in PICU [12]. Mean blood pressure is one of the variables in APACHE score, which predicts the probability of death [24]. Capillary refill time is one simple bedside tool that is used in the assessment of circulation in a sick child presenting to the hospital for treatment [25]. Capillary refill time is one of the variables of ETAT scoring which is developed for rapid initiation of appropriate treatment [7]. Tibby SM has concluded that delayed capillary refill time is a useful marker of hypovolemia and probably poor myocardial function [26].

Mc Carthy P L et al concluded $\mathrm{SPO}_{2}$ as the fifth vital sign in pediatric emergency room [26]. Study done by Onyngo FE showed association of hypoxemia with mortality. Altered sensorium level is present in hypoxaemia and poor circulation. Assessment of sensorium is an important variable in the assessment of overall wellbeing in children.

On univariate Analysis, temperature and heart rate were not significantly associated with outcome. Probably recording the temperature of both axillas \& taking the mean would have been more useful [9]. The respiratory rate, blood Pressure, $\mathrm{SPO}_{2}$, capillary refill Time \& sensorium in our study was significantly associated 
with outcome. Study done by Kumar et al based on same physical variables concluded that heart rate and respiratory rate were not associated significantly with survival status, while rest of them had significant association [1] .We have found on ROC Curve Analysis, that SICK scoring system has predictive ability in assessing the outcome in this study done in tertiary care center in south India. We further tried to assess the validity of SICK score based on the predominant system involved at the time of presentation and found the score to be valid and no significant variation in the score, cut off and area under curve in different systems.

\section{Conclusion}

SICK score uses only physical criteria without laboratory variables, so can prioritize sick children at presentation with no special training needed for implementation. On this basis we propose it to be a cost effective scoring system for prioritizing sick children in resource poor settings

Funding: Nil, Conflict of interest: None initiated, Perission from IRB: Yes

\section{References}

1. Kumar N, Thomas N,SinghalD,PuliyelJM,Sreenivas $\mathrm{V}$ :Triage score for severity of illness:IndianPed 2003,40:204-10.https://www.ncbi.nlm.nih.gov/ pubmed / 12657751

2. Robertson M A,Molyneux E M,Triage in developing world-can it be done? Arch Dis Child 2001,85:208-13. adc.bmj.com/content/88/7/563.full.pdf.

3. PollackMM,RuttimannUE,GetsonPR.The Pediatric risk of mortality(PRISM) Score.Crit Care Med 1988,16:1110-16. https:/www.ncbi.nlm.nih.gov/pmc / articles /PMC1763066/pdf/v087p00421.pdf

4. ChakrabartyA, HalsteadR, SahniM, RangasamyJ, PuliyelA ,SreenivasV,GreenDA,PuliyelJM:Validation of "Signs of inflammation in children that kill"( SICK) Score for noninvasive assessment of severity of illness:Italian Journal of Pediatrics 2010,36:35. https://ijponline.biomedcentral.com/articles/10.1186/18 24-7288-36-35

5. ShannF,PearsonG,SlaterA,WilkinsonK. Pediatric Index of Mortality(PIM):A mortality prediction model in intensive care.Intensive Care Med 1997:23:201-07. https://www.ncbi.nlm.nih.gov/pubmed/9069007
6. Robertson M A, Molyneux E M. Description of cause of serious illness and outcome in patients identified using ETAT guidelines in Urban Malawi. Arch Dis Child 2001; 85:214-17. archdischild-2013-305469v1

7. Gove S, Tamburlini G, Molyneux E, Whitesell P, and Campbell H. Development and technical basis of simplified guidelines for emergency triage assessment and treatment in developing countries.Arch Dis Child. 1999 December; 81(6): 473-477. www.ncbi. nlm.nih. gov > Journal List > Arch Dis Child > v.81(6); 1999 Dec.

8. Thompson N, Coad N, Harnden A, Mayon white R. How well do vital signs identify children with serious infections in Pediatric Emergency Care? Arch Dis Child 2009 Nov; 94(11): 888-9. https://www.ncbi.nlm.nih. gov/pubmed/19608555

9. Bhal S,TyagiV,KumarN,Sreenivas V PuliyelJM;Signs of inflammation that can kill(SICK)score:Preliminary prospective validation of a non invasive $\mathrm{m} 3859$;year $=2006$; measure of severity of illness. JPostgrad Med2006;52:52:102-5. www. jpgmonline. $\mathrm{com} /$ article.asp?issn $=0022$

10. Mackway-Jones K: Emergency triage, Manchester Triage group; London BMJ Publishing group; 1997 (Pub Med). www.bmj.com/content/337/bmj.a1501

11. Pal Comstedt, Merete Storgard and AnnMarie T Lassen. Systemic Inflammatory Response Syndrome in acutely hospitalized patients, a cohort study: Scandinavian Journal of Trauma, Resuscitation \& Emerg Medicine 2009: 17-27. https://sjtrem. biomedcentral.com/articles/10.1186/1757-7241-17-67

12. Slater A, Shann F. Suitability of Pediatric Index of Mortality (PIM), PIM2, PRISM \& PRISM III for monitoring the quality of pediatric intensive care in Australia and Newzealand Pediatr crit care Med 2004 Sep; 5(5): 447-54. https://www.ncbi.nlm.nih. gov/ pubmed/ 15329160

13. Wells M, Reira-Fanego JF, Luyt DK, Dance M, Lipman J. Poor discriminatory performance of the pediatric risk of mortality (PRISM) score in South African Intensive Care Unit. Crit care med 1997; 25: 716-7. https: //www. ncbi. nlm.nih.gov/pubmed/ 8797623 
14. John M, Goddard FC. Pediatric Risk of Mortality (PRISM) score overestimates the severity of illness in infants. Crit Care Med 1992; 20:1662-65.

15. Steven D Burdette, Miguel A Parilo, Lewis J Kaplan, Heatherlee Bailej. Systemic inflammatory response syndrome. E Medicine specialties (updated Apr.15, 2010) URL:http://www. emedicine. com /med/topic2227.html.

16. Sayagama A, Aikawa N. Evaluation of systemic inflammatory response syndrome criteria as a predictor of mortality in patients transported by ambulance Keio $\mathrm{J}$ Med 1999 Mar; 48(1):28-37. www.ncbi.nlm.nih. gov/ pubmed

17. Maria Antonette Enrione and Keith R Powell. Sepsis, septic shock and systemic inflammatory response syndrome. In; Behrman, Kliegman, Jension. Nelson Textbook of pediatrics, Saunders Company, Philadelphia $18^{\text {th }}$ ed (Published in India by Elsevier, a division of India private limited) 2008: 1094-99.

18. Mackway Jones K, Molyneux E, Philips B, Weeteskas. Structured approach to the seriously ill child. In: Advanced pediatric life support. Online library.wiley.com/doi/10.1002/9781119241225.ch4

19. The practical approach. BMJ Books, $3^{\text {rd }}$ Edition. Panther publishers Bangalore 2001;pp: 71-78.

20. Mc Carthy P L, Grundy G W. Bacteremia in children: an outpatient clinical review. Pediatrics 1976; 57:861-868. jamanetwork.com/journals/jamapediatrics/ fullarticle/514274.
21. Campbell H, Byass P, Greenwood B M. Simple clinical signs for diagnosis of acute lower respiratory infections. Lancet. 1988; 2: 742-743. https://www. ncbi.nlm.nih.gov > NCBI > Literature > Pub Med Central (PMC)

22. Cherian T, John T J, Simoes E, Steinhoff M C, John $\mathrm{M}$, Evaluation of simple clinical signs for diagnosis of acute lower respiration tract infection. Lancet. 1988; 2: 125-128. https: //www.ncbi.nlm.nih.gov >NCBI > Literature > PubMed Central (PMC)

23. Horari M, Shann F, Spooner V, Meisner S, Carney M, Decampo J. Clinical signs of pneumonia in children. Lancet. 1991; 338: 928-930. thelancet.com/journals/ laninf/article/PIIS1473-3099(15)70017-4

24. Kevin Gunning, Kathy Rowan. Outcome data and scoring systems. BMJ 1999; 319: 241-44. www.bmj. com/ rapid.../2011/.../mpm-preferable-apache-intensivecare-scoring-system

25. Circulation, Chapter 7, Facility based Integrated management of Neonatal and childhood illness (F.IMNCI) Participants Manual; 28-34. www. health.mp. gov.in/manuals/STG-2016.pdf

26. Tibby S, Murdoch I A. Monitoring cardiac function in intensive care. Arch Dis Child 2003; 88: 46-52. https://www.ncbi.nlm.nih.gov/pubmed/12495962

27. Onyngo F E, Steinhoff M C, Wafula E M, Ariua S W, Musia J, Kitonyi J. Hypoxemia in young Kenyan children with acute lower respiratory tract infection. BMJ 1999; 306: 612-615.

\section{How to cite this article?}

Babji S.K, Bala gopal M, Sharma N.A, Sivaguru C. Validation of SICK score - A severity of illness scoring system at a tertiary care centre in Puducherry. J PediatrRes.2017;4(03):231-239.doi:10. 17511/ijpr.2017.03.06. 